\title{
Commentaries
}

\section{Is bacterial ash the flash that ignites NASH?}

As obesity creeps in epidemic proportions through increasingly inert and affluent "developed" societies, metabolic imbalance has become the commonest cause of liver disease. Fatty liver, although itself benign, predisposes the liver to NASH or non-alcoholic steatohepatitis, a chronic disorder characterised by steatosis, mixed cell type inflammation, focal hepatocyte degeneration, and perivenular or pericellular fibrosis. ${ }^{12} \mathrm{NASH}$ is slowly progressive, occasionally resulting in cirrhosis with the potentially fatal complications of portal hypertension, liver failure, and hepatocellular carcinoma. ${ }^{2}$

Most cases of NASH appear to have a multifactorial aetiopathogenesis. The predisposing factors include obesity, type II diabetes, insulin resistance, hypertriglyceridaemia, and rapid weight loss, each of which can cause hepatic steatosis. The trigger that sets off injury and inflammation against this background of oxidisable fatty acid excess, and the mechanisms that perpetuate steatohepatitis and fibrogenesis are less clear. ${ }^{4} \mathrm{~A}$ focus of recent experimental studies has been on biochemical processes that reduce oxygen to reactive oxygen species (ROS); these can potentially damage tissues by the process of oxidative stress. Oxidative stress also increases expression of cellular adhesion molecules, and secretion of chemokines and cytokines, thereby initiating the recruitment of an hepatic inflammatory response. ${ }^{56}$

Weltman and colleagues demonstrated that cytochrome P450 (CYP)2E1 is over expressed in the livers of patients with $\mathrm{NASH}^{7}$ as well as in a model of steatohepatitis induced by feeding rats a high fat, methionine and choline deficient (MCD) diet. ${ }^{8}$ Leclercq and colleagues then showed that CYP2E1 catalyses lipid peroxidation in the murine MCD dietary model, ${ }^{9}$ contributing to profound oxidative stress. Alternatively, in Cyp2e1 nullizygous mice, CYP4A enzymes are upregulated and function as alternative catalysts of microsomal lipoperoxidation in experimental NASH. ${ }^{9}$ Other studies have implicated activation of the peroxisome proliferator activated receptor $\alpha(\operatorname{PPAR} \alpha)$ in a pathway leading to $\mathrm{NASH}^{10}$ Activated PPAR $\alpha$ is a transcription factor that governs expression of the peroxisomal and microsomal (via CYP4A) pathways of lipid oxidation, with consequent over production of ROS.

As opposed to the possible primary role of "biochemical oxidative stress", it is possible that the inflammatory response can be the primary mediator of liver cell injury in steatohepatitis. This is a favoured mechanism in alcoholic hepatitis in which gut derived endotoxin appears to incite necroinflammatory change, ${ }^{6}$ even in the absence of CYP2E $1 .{ }^{11}$ Endotoxin is a potent releaser of proinflammatory and cytotoxic cytokines such as tumour necrosis factor $\alpha(\mathrm{TNF}-\alpha)$. The cell types involved include activated macrophages, including Kupffer cells, lymphocytes, and neutrophils. In alcoholic hepatitis, ${ }^{6}$ and potentially in $\mathrm{NASH}$, such cell types could be recruited to the liver where they not only contribute further to oxidative stress by release of ROS and nitroradicals ${ }^{12}$ but also release cytokines that contribute to liver cell injury and hepatic fibrogenesis.
The distinction between NASH and steatosis without inflammation, injury, or fibrosis is never clearer than in the ob/ob leptin deficient mouse. This animal exhibits exogenous obesity, diabetes, and a steatotic liver that resembles Swiss cheese without the rind (there is no fibrosis!). In ob/ob mice, as well as in other rodent models of uncomplicated hepatic steatosis, administration of endotoxin provokes liver inflammation with focal hepatocyte injury, probably by releasing a shower of TNF- $\alpha$ from Kupffer cells, macrophages, and other cell types. ${ }^{13}{ }^{14}$

Until now it has been unclear if these experimental findings have any relevance to the pathogenesis of NASH in the human liver. An earlier clue came from the severe steatohepatitis, occasionally fatal, that occurred after jejunoileal bypass (JIB) surgery for obesity. ${ }^{15}$ NASH has also been described in adults during total parenteral nutrition, ${ }^{16}$ and in a case of multiple jejunal diverticulae with bacterial overgrowth of the small intestine ${ }^{17}$ In these conditions, metronidazole therapy reduced liver injury in some cases, although use of tetracycline was less effective. ${ }^{17}$ Furthermore, in a rat model of JIB liver disease, resection of the redundant intestinal loop with its bacterial flora, or treatment with metronidazole or tetracycline, improved liver disease. ${ }^{18}$ The mechanism appeared to be improved absorption of micronutrients, including vitamin $\mathrm{E}$, a critical membrane antioxidant, and possibly essential amino acids required to synthesise the principal cellular antioxidant, glutathione. ${ }^{18}$

Despite the dire consequences of severe NASH after earlier JIB, there has seemed little reason to suspect that the intestinal flora has much to do with the usually insidious process of NASH. The report from Adelaide by Wigg and colleagues published in this issue of Gut challenges this complacency (see page 206). ${ }^{19}$ The investigators studied small intestinal function and serum TNF- $\alpha$ levels in 22 patients with NASH and 23 controls. Using a combined ${ }^{14} \mathrm{C}$-D-xylose and lactulose breath test to define small intestinal bacterial overgrowth, they found that half of the patients with NASH had evidence of bacterial overgrowth compared with $22 \%$ of controls. Furthermore, serum TNF- $\alpha$ levels were significantly increased in the NASH group compared with controls. These findings entice us to consider the plausible proposal that gut derived bacterial toxins could be involved in triggering liver injury in the context of hepatic steatosis - or to paraphrase the title of this commentary: Do gut bugs trash the stash of liver hash into NASH?

Before accepting this contribution as a real advance towards understanding the pathogenesis of NASH, the limitations of the study should be considered. The patient groups were small and imperfectly matched in some critical variables, such as obesity, diabetes, and hypertriglyceridaemia. A positive ${ }^{14} \mathrm{C}-\mathrm{D}$-xylose and lactulose breath test was strongly associated with diabetes, as might be expected from the decreased gut motility that commonly results from diabetic neuropathy (albeit that an impressive slowing of gut transit time could not be demonstrated). Could this have biased the findings so that the apparent association with NASH is spurious? To answer this, further studies comparing diabetics with and without NASH are required.

In the Adelaide study, the definition of small intestinal bacterial overgrowth rested on a single parameter, and the apparent high frequency of abnormalities in relatively healthy controls begs the issue of test reliability to define an 
apparently subtle form of bacterial overgrowth. The definition $\mathrm{NASH}$ may also have been inadequate ${ }^{1}$ : it requires histological assessment because liver test abnormalities and hepatic imaging do not reliably discriminate between uncomplicated steatosis and NASH. In this study, three of the NASH patients (and all of the controls) were not subjected to liver biopsy, leaving open the opportunity for misclassification of cases.

Despite the finding that TNF- $\alpha$ levels were increased in patients with NASH, Wigg et al were unable to demonstrate either a "leaky" infected small intestine (as measured by the lactulose-rhamnose sugar test) or endotoxaemia. ${ }^{19}$ At first glance, this seems counterintuitive for the proposal that bacterial overgrowth of the small intestine plays a pathogenic role in NASH. Some plausible explanations for the paradox were suggested: limitations of the limulus assay, binding of endotoxin to plasma proteins, and systemic levels may not reflect portal endotoxaemia. Furthermore, other bacterial products such as peptidoglycan-polysaccharide polymers rather than endotoxin could stimulate release of TNF- $\alpha$. The latter concept is particularly cogent because Bacteroides species rather than aerobic Gram negative bacteria such as Escherichia coli, the source of endotoxin, appear to be implicated in the pathogenesis of small intestinal bacterial overgrowth. ${ }^{20}$ Measurement of peptidoglycanpolysaccharide polymers in patients with NASH would be an interesting direction of investigation in the future.

In the Adelaide study, there was no relationship between body mass index and serum TNF- $\alpha$ levels, but a link between obesity and raised serum TNF- $\alpha$ has been described by others. ${ }^{21}$ Yang and colleagues noted that after endotoxin administration in leptin deficient $o b / o b$ mice, hepatic induction of IFN- $\gamma$ is increased whereas IL-10 induction is inhibited. ${ }^{13}$ IFN- $\gamma$ increases hepatocyte sensitivity to TNF- $\alpha$ while IL-10 appears to inhibit the tissue response to TNF- $\alpha$. These findings were interpreted as indicating possible macrophage dysfunction in obesity in a way that could promote steatohepatitis by sensitising hepatocytes to endotoxin. ${ }^{13}$ Guebe-Xabier et al have also shown that $o b / o b$ mice have a selective reduction of hepatic $\mathrm{CD}^{+} \mathrm{NK} \mathrm{T}$ cells, and this is associated with and possibly mediated by upregulation of IL-18 and IL-12. ${ }^{14}$ Whether these abnormalities of lymphocyte populations and cytokine responses are due to obesity per se or to leptin deficiency (which is not a feature of human obesity) remains to be determined.

NASH can be regarded as the hepatic consequence of the metabolic syndrome (central obesity, insulin resistance, type II diabetes, arterial hypertension, hyperlipidaemia). ${ }^{22}{ }^{23}$ Attention has shifted from the reasons for steatosis, much of which is benign or resolves in the advanced stages of cirrhosis, to the mechanisms for hepatocellular injury, inflammation, and fibrosis. ${ }^{413}$ The findings reported by Wigg et al, while not definitive, may provide a new clue to the importance of cytokines in mediating liver cell injury in NASH. Whether the release of TNF- $\alpha$ is a consequence of small intestinal bacterial overgrowth, obesity, or oxidative stress will require further study.

G C FARRELL

Storr Liver Unit, Westmead Millennium Institute,

University of Sydney at Westmead Hospital,

Westmead, NSW 2145, Australia

geoff_farrell@wmi.usyd.edu.au

1 Brunt EM, Janney CG, Di Bisceglie AM, et al. Nonalcoholic steatohepatitis: a proposal for grading and staging the histological lesions. Am f Gastroenterol 1999; 94:2467-74.

2 James O, Day C. Non-alcoholic steatohepatitis: another disease of affluence. Lancet 1999;353:1634-6.

3 Matteoni CA, Younossi ZM, Gramlich T, et al. Nonalcoholic fatty liver disease: A spectrum of clinical and pathological severity. Gastroenterology 1999;116:1413-19.

4 Day CP, James OFW. Steatohepatitis: a tale of two "hits"? Gastroenterology 1998;114:842-4.

5 Lee KS, Buck M, Houglam K, et al. Activation of hepatic stellate cells by TGF alpha and collagen type 1 is mediated by oxidative stress through TGF alpha and collagen type 1 is mediated by
c-myb expression. 7 Clin Invest 1995;96:2461-8.

6 McClain CJ, Barve S, Deaciuc I, et al. Cytokines in alcoholic liver disease. Semin Liver Dis 1999;19:205-19.

7 Weltman MD, Farrell GC, Hall P, et al. Hepatic cytochrome P450 2E1 is increased in patients with nonalcoholic steatohepatitis. Hepatology 1998;27: 128-33.

8 Weltman MD, Farrell GC, Liddle C. Increased hepatic CYP2E1 expression in rat model of hepatic steatosis with inflammation. Gastroenterology 1996; 111:1645-53.

9 Leclercq IA, Farrell GC, Field J, et al. CYP2E1 and CYP4A as microsomal catalysts of lipid peroxides in murine nonalcoholic steatohepatitis. $7 \mathrm{Clin}$ Invest 2000;105:1067-75.

10 Fan C-Y, Pan J, Usuda N, et al. Steatohepatitis, spontaneous peroxisomal proliferation and liver tumors in mice lacking peroxisomal fatty acyl-CoA oxidase. Implications for peroxisomal proliferator-activated receptor $\alpha$ natural ligand metabolism. $\mathcal{F}$ Biol Chem 1998;273:15639-45.

11 Kono H, Bradford BU, Yin M, et al. CYP2E1 is not involved in early alcohol-induced liver injury. Am f Physiol 1999;277:G1259-67.

12 Zhang C, Walker LM, Hinson JA, et al. Oxidant stress in rat liver after lipopolysaccharide administration: effect of inducible nitric-oxide synthase inhibition. F Pharm Exp Ther 2000;293:968-72.

13 Yang SQ, Lin HZ, Lane MD, et al. Obesity increases sensitivity to endotoxin liver injury: implications for the pathogenesis of steatohepatitis. Proc Natl Acad Sci USA 1997;94:2557-62.

14 Guebre-Xabier M, Yang SQ, Lin HZ, et al. Altered hepatic lymphocyte subpopulations in obesity-related murine fatty livers: potential mechanism for sensitization to liver damage. Hepatology 2000;31:633-40.

15 Yost RL, Duerson MC, Russell WL, et al. Doxycycline in the prevention of hepatic dysfunction: an evaluation of its use following jejunoileal bypass in humans. Arch Surg 1979;114:931-4.

16 Quigley EM, Marsh MN, Shaffer JL, et al. Hepatobiliary complications of total parenteral nutrition. Gastroenterology 1993;104:286-301.

17 Nazim M, Stamp G, Hodgson HJ. Non-alcoholic steatohepatitis associated with small intestinal diverticulosis and bacterial overgrowth. Hepatogastroenterology 1989;36:349-51.

18 Vanderhoof JA, Metz MJ, Tuma DJ, et al. Effect of improved absorption on development of jejunileal bypass-induced liver dysfunction in rats. Dig Dis Sci 1980;25:581-6.

19 Wigg AJ, Roberts-Thomson IC, Dymock RB, et al. The role of small intestinal bacterial overgrowth, intestinal permeability, endotoxaemia, and tumour necrosis factor $\alpha$ in the pathogenesis of non-alcoholic steatohepatitis. Gut 2001;48:206-11.

20 Lichtman SN, Keku J, Schwab JH, et al. Hepatic injury associated with small bowel bacterial overgrowth in rats is prevented by metronidazole and tetracycline. Gastroenterology 1991;100:513-19.

21 Hotamisligil GH, Spiegelman BM. Tumor necrosis factor- $\alpha$. A key component of the obesity-diabetes link. Diabetes 1994;43:1271-8.

22 Marceau P, Biron S, Hould F-S, et al. Liver pathology and the metabolic syndrome X in severe obesity. F Clin Endocrinol Metab 1999;84:1513-17.

23 Cortez-Pinto $\mathrm{H}$, Camilo ME, Baptista A, et al. Non-alcoholic fatty liver: another feature of the metabolic syndrome? Clin Nutr 1999;18:353-8.
The development of hepatocellular carcinoma (HCC) constitutes a frequent event during the evolution of patients with liver cirrhosis ( $3-5 \%$ annual incidence rate) and constitutes their main cause of death. ${ }^{1}$ Survival is related to tumour stage at diagnosis and to the degree of impairment of liver function. Recent data have shown that survival after diagnosis is not as poor as reported years ago. ${ }^{2}$ This is due both to advances in diagnosis even in the absence of effective treatment (lead time bias) and to the application of curative treatments (surgical resection, liver transplantation, and percutaneous ablation). ${ }^{2}$ These offer the only chance of cure but their applicability and long term success with five year survival exceeding $50 \%$ require the detection of HCC at an early stage, including patients with solitary nodules $\leqslant 5 \mathrm{~cm}$ or up to three nodules each $\leqslant 3 \mathrm{~cm} .{ }^{2-5}$ In contrast, large/multifocal tumours are less likely to benefit from curative approaches and here three year survival falls below $50 \%$ regardless of treatment. ${ }^{2}$ The need for detection of HCC at an early stage has prompted 
surveillance programmes for patients with cirrhosis. HCC has most requisites for such a policy ${ }^{6}$ : the population at risk is known, the disease is highly prevalent, it has a high mortality, and effective screening tests are available and acceptable. However, other conditions are not yet met: the recall policy on raising suspicion is not well defined and, unfortunately, there is no unequivocal proof that treatment improves survival. Radical therapies have never been evaluated in randomised controlled trials but are widely considered "effective" and assumed to improve survival. . $^{3-5}$ In contrast, randomised controlled trials assessing palliative treatments (that is, chemoembolisation) have shown negative results. ${ }^{2}$ Accordingly, the usefulness of surveillance programmes in cirrhotics is still controversial, leading to the suggestion that they provide a minor benefit in terms of efficacy (years of life saved) and cost effectiveness. The only method to clarify this issue would be to design a randomised controlled trial comparing surveillance versus non-surveillance in a large series of cirrhotics who would be treated if diagnosed with HCC. Such an investigation would be ethically questionable and in addition, almost unfeasible. Ultrasound examination is commonly used for evaluation of cirrhotics, irrespective of the type of symptoms and this would "contaminate" the control arm.

In the absence of randomised controlled trials, how may we estimate the benefits of surveillance? Two approaches are proposed: to conduct follow up investigations or to perform decision-analytical studies using assumptions reported in clinical studies. ${ }^{7}$ Bolondi and colleagues ${ }^{8}$ conducted a follow up study, reported in this issue of Gut, recruiting a large series of cirrhotics and applying the most common surveillance policy: ultrasound and $\alpha$ fetoprotein determinations every six months (see page 251). On suspicion, they followed a predefined recall policy to determine the diagnosis and select the most suitable therapy aiming to offer all radical options to patients with early HCC. The outcome of patients under surveillance was compared with that of patients referred to hospital for HCC that was incidentally detected outside their programme. This comparison has allowed a rough estimation of the surveillance benefits but detection of patients with asymptomatic small solitary tumours outside surveillance suggests potential "contamination" of the control group by uncontrolled surveillance within the community physicians who thereafter refer patients with suspected HCC to the tertiary hospital for evaluation and treatment.

As surveillance is aimed at reducing disease specific mortality, comparison of long term survival between both cohorts is crucial. Unfortunately, the difference in survival was significant but not impressive $(45 \% v 32 \%$ at three years). This may reflect both a lead time phenomenon and a real impact of treatment on survival. Interestingly, the applicability of radical therapies was not significantly different between the two cohorts $(69 \% v 54 \%)$, although liver transplantation was more frequently applied in the surveillance cohort. Nevertheless, multivariate analysis identified liver function and tumour stage as survival predictors. Tumour stage may be a surrogate of surveillance and thus surveillance may prompt earlier HCC detection not allowing a better therapeutic approach, and this would prevent a marked impact on survival. In addition to this clinical output, Bolondi et al showed that the cost per year of life saved was above US\$100 000, a value largely exceeding the cut off accepted for surveillance by policy makers and health providers. ${ }^{9}$

Do these findings imply that the study is not relevant? The answer is no. Evaluation of surveillance for HCC requires several clinical studies with different designs in different settings. Bolondi and colleagues ${ }^{8}$ describe the outcome of a hospital based programme including all types of cirrhotics and it may be that the clinical impact would be higher in a community based programme. In the latter, the risk of HCC might be lower because of better liver function (the study confirms age, advanced liver disease, and increased $\alpha$ fetoprotein concentrations as the main HCC predictors within cirrhosis) but the applicability of liver resection or transplantation may be increased. Thus a relevant increase in survival could be attained. On the other hand, the cost of each detected HCC in low risk individuals may also rise, and perhaps the unacceptable cost effectiveness ratio would not be modified. Another approach is more intense surveillance (that is, every three months) or the use of different tools. The results of these awaited studies will provide the assumptions to be used for estimation of the benefits and cost effectiveness of surveillance in different scenarios by using statistical techniques such as the Markov model.

Until these data become available, the debate will persist and surveillance will be initiated in cirrhotics, even without evidenced based data. However, this approach does have positive benefits. The diagnosis of patients at a nonadvanced stages prompts further refinement of treatments with progressive improvement in long term outcomes. In addition, in the era of genetic profiling, careful recruitment of both clinical and biological data within surveillance will surely introduce molecular concepts into clinical practice. Ultimately, this research will change our understanding of the disease and help us to identify new targets for both prevention and treatment.

J BRUIX

J M LLOVET

BCLC Group, Liver Unit, Hospital Clínic, IDIBAPS, University of Barcelona, Catalonia, Spain

Correspondence to: Dr J Bruix, BCLC Group, Liver Unit, Hospital Clínic, University of Barcelona, Villarroel 170, 08036-Barcelona, Catalonia, Spain. jbruix@clinic.ub.es

1 Colombo M, De Franchis R, Del Ninno E, et al. Hepatocellular carcinoma in Italian patients with cirrhosis. N Engl F Med 1991;325:675-80. 2 Llovet JM, Brú C, Bruix J. Prognosis of hepatocellular carcinoma: The BCLC staging classification. Semin Liver Dis 1999;19:329-38.

3 Mazzaferro V, Regalia E, Doci R, et al. Liver transplantation for the treatment of small hepatocellular carcinomas in patients with cirrhosis. $N$ Engl F Med 1996;334:693-9.

4 Llovet JM, Fuster J, Bruix J. Intention-to-treat analysis of surgical treatment for early hepatocellular carcinoma: resection versus transplantation. Hepatology 1999;30:1434-40.

5 Bismuth H, Majno PE, Adam R. Liver transplantation for hepatocellular carcinoma. Semin Liver Dis 1999;19:311-22.

6 Collier J, Sherman M. Screening for hepatocellular carcinoma. Hepatology 1998;27:273-8.

7 Sarasin F, Giostra E, Hadengue A. Cost-effectiveness of screening for detection of small hepatocellular carcinoma in western patients with Child-Pugh tion of small hepatocellular carcinoma in wester
class A cirrhosis. Am $7 \mathrm{Med}$ 1996;171:422-34.

8 Bolondi L, Sofia S, Siringo S, et al. Surveillance programme of cirrhotic patients for early diagnosis and treatment of hepatocellular carcinoma: a cost effectiveness analysis. Gut 2001;48:251-9.

9 Laupacis A, Feeny D, Detsky AS, et al. How attractive does a new technology have to be to warrant adoption and utilization? Tentative guidelines for using clinical and economic evaluations CMAf 1992;146:473-81. 


\section{Endoscopic mucosal resection for early gastric cancer}

The paper by Ono and colleagues ${ }^{1}$ in this issue of Gut documents the remarkable results achieved at the Tokyo National Cancer Centre Hospital (NCCH) with endoscopic management of early gastric cancer (EGC) (see page 225). EGCs constitute a much higher proportion of the total number of gastric cancers in Japan than is the case in the West. There are a number of reasons for this: firstly, many asymptomatic subjects in Japan are screened for gastric cancer (usually by barium radiology, followed, if necessary, by endoscopy); secondly, Japanese diagnostic gastroscopy is a much more careful procedure than the "smash and grab" style of endoscopy which is typical in this country: the stomach is inflated to a greater degree, indigo carmine dye spraying is used to examine any suspicious area, and simethicone (to eliminate bubbles and froth) and hyoscine (to paralyse the stomach) are used routinely. There is some evidence that early lesions are missed in the UK: a recent audit in one centre showed that 11 of 81 patients presenting with advanced gastric cancer had undergone endoscopy within the previous two years. $^{2}$

Is EGC in Japan the same disease as EGC in the West? A recent analysis of published data from Japan and the rest of the world ${ }^{3}$ concluded that histological type, macroscopic appearance, degree of invasion, and frequency of lymph node metastases are the same in Europe as Japan. Japanese classification of tumour invasion, as Ono et al have pointed out, differs, with lesions classified as "high grade dysplasia" in the West called "intramucosal carcinoma" in Japan. A new terminology-the Vienna classification of gastrointestinal neoplasia ${ }^{4}$ - has been proposed in an effort to overcome this discrepancy.

Endoscopic mucosal resection (EMR) for EGCs confined to the mucosa seems a very attractive alternative to surgery. Ono et al wish to "promote its use around the world" and their paper is a powerful argument in favour of EMR. Could EMR become a standard therapy for mucosal EGC in this country? EMR demands a high level of endoscopic expertise, as well as a cooperative patient. I have had the opportunity to examine the case from both sides, having visited the $\mathrm{NCCH}$ and having played host to endoscopists from the NCCH who worked in our unit over the past few years. I have personally witnessed an EMR at the NCCH which took over three hours to complete (the lesion was large); the patient was lightly sedated with midazolam and remained cooperative throughout. Our patients are less likely to tolerate these procedures as well as the Japanese. Endoscopists from the NCCH carried out a number of EMRs for EGC in our unit in April of this year as part of a "live" demonstration in a course on endoscopic management of early gastrointestinal cancer, and all patients required a general anaesthetic. As well as anaesthetic input, EMR requires special equipment and a dual channel therapeutic gastroscope. Although the technique of "strip biopsy" is relatively straightforward, and suitable for smaller lesions, it is more likely to result in resection in multiple fragments which makes histological evaluation of resection much more difficult. (In this series, completeness of resection could not be evaluated in $20 \%$.)

The NCCH endoscopists now prefer the newer technique employing the insulation tipped diathermic knife
(IT knife) which is not commercially available in the UK. This technique is technically much more demanding and time consuming but can be used to resect much larger lesions: I have seen an $8 \mathrm{~cm}$ EGC resected with this technique (the patient was unfit for surgery). EMR is not generally suitable however for large lesions: in this series, complete resection was achieved in only $38 \%$ of lesions $>3 \mathrm{~cm}$.

Alternatives to strip biopsy have been described by other Japanese groups: these include aspiration mucosectomy ${ }^{5}$ and EMR using a ligating device. ${ }^{6}$ Proponents of aspiration mucosectomy claim it is technically easier than strip biopsy.

Japanese endoscopists are expert in assessing depth of invasion of gastric cancers purely on the basis of endoscopic features. ${ }^{7}$ Some criteria, such as size and presence of ulceration, are straightforward, but others, such as the macroscopic configuration (superficial, elevated, depressed, etc) and mucosal fold pattern, are more subjective and may present difficulty for western endoscopists. Ono et al "do not use endoscopic ultrasonography (EUS) routinely, as it is not sensitive enough to evaluate minute invasion to the submucosa." Nevertheless, $15 \%$ of resected lesions showed submucosal invasion. I suspect that Western endoscopists would opt for routine use of EUS before embarking on EMR.

Endoscopists inexperienced with EMR will have concerns about complications of bleeding and haemorrhage. Ono et al had a relatively high incidence of perforation at $5 \%$ (the risk of bleeding is not stated). Remarkably, most of these perforations were managed by endoscopic clipping. Japan has an entirely different medical culture to the UK: litigation and complaints are uncommon, and advice given by doctors is usually accepted without question. British endoscopists will be warier of new techniques with potentially serious complications. In this brave new world of clinical governance, who is to determine what an acceptable complication rate should be for a new procedure? The current political climate in the NHS discourages the development of techniques such as EMR. Few centres in this country are likely to diagnose enough EGCs to become expert in EMR, and it would be desirable, therefore, for this technique to become concentrated in a few specialised centres.

Patients with EGC can expect a five year survival rate in excess of $90 \%$ with surgery ${ }^{8}$; why then should we in the West embrace EMR? Ono et al have pointed out a number of compelling reasons: firstly, EGCs fulfilling the criteria for EMR have a lower risk of lymph node metastasis $(0.36 \%)$ than the mortality rate from surgery for EGC at their centre $(0.5 \%)$; secondly, EMR is considerably cheaper compared with conventional surgery; and thirdly, there were no gastric cancer related deaths in this series during a median follow up period of 38 months. Surgery will continue to play a key role in the management of EGC: despite the success of endoscopic therapy, most EGCs at the Tokyo NCCH are still treated surgically.

EMR in this country is likely to develop initially as therapy for EGC in frail patients who would pose a high risk for radical surgery; with increasing experience, we can aspire to the outcomes achieved by the Japanese.

S O'MAHONY

Centre for Digestive Diseases, Leeds General Infirmary,

Great George St, Leeds LS1 3EX, UK

seamuso@ulth.northy.nhs.uk

1 Ono $\mathrm{H}$, Kondo H, Gotoda T, et al. Endoscopic mucosal resection for treatment of early gastric cancer. Gut 2001;48:225-9. 
2 Suvakovic Z, Bramble MG, Jones R, et al. Improving the detection rate of early gastric cancer requires more than open access endoscopy: a five year early gastric cancer requires
study. Gut 1997;41:308-13.

3 Everett SM, Axon ATR. Early gastric cancer in Europe. Gut 1997;41:14250 .

4 Schlemper RJ, Riddell RH, Kato Y, et al. The Vienna classification of gastrointestinal neoplasia. Gut 2000;47:251-5.

5 Tanabe S, Koizumi W, Kokutou M, et al. Usefulness of aspiration mucosectomy as compared with strip biopsy for the treatment of gastric mucosal cancer. Gastrointest Endosc 1999;50:819-22.
6 Suzuki Y, Hiraishi H, Kanke K, et al. Treatment of gastric tumors by endoscopic mucosal resection with a ligating device. Gastroinest Endosc 1999;49: scopic m.

7 Sano T, Okuyama Y, Kobori O, et al. Early gastric cancer: endoscopic diagnosis of depth of invasion. Dig Dis Sci 1990;35:1340-4.

8 Sue-Ling HM, Martin I, Griffith J, et al. Early gastric cancer: 46 cases treated in one surgical department. Gut 1992;33:1318-22.

9 Yamazaki H, Oshima A, Murakami R, et al. A long-term follow-up study of patients with gastric cancer detected by mass screening. Cancer 1989;63: 613-17. 